\title{
Energy-Aware, Collaborative Tracking with Ad-Hoc Wireless Sensor Networks
}

\author{
S. Balasubramanian, S.K. Jayaweera, and K.R. Namuduri \\ Department of Electrical and Computer Engineering, College of Engineering
}

\begin{abstract}
An energy aware, collaborative tracking algorithm is proposed for ad-hoc wireless sensor networks consisting of randomly distributed low-end sensors and a high-end data gathering node which is geometrically located at the center of each cluster. The collaborative tracking algorithm is implemented distributively by passing sensing and computation operations from one cluster to another. The network lifetime is maximized by choosing active sensors according to an energy-based cost function. Simulation results for both single and multiple simultaneously active sensors have also been preformed. Performance is evaluated based on both tracking error and energy consumption of the whole network.
\end{abstract}

\section{Introduction}

An ad-hoc wireless sensor network consists of a set of randomly distributed sensors that communicate with each other over a radio link. In this paper, we focus on such an ad-hoc wireless sensor network deployed for collaborative target tracking. The sensors in this network are assumed to have finite sensing radii and hence the network can conveniently be divided into multiple clusters. An active cluster in such a network is defined as one in which a moving target of interest is currently present. For simplicity, the moving target is assumed to be on the same plane on which the sensors are located and only an active sensor is expected to detect and track the target. All other sensors will be in an idle mode so as to save the energy [1].

Most often sensor nodes in an ad-hoc wireless sensor network are battery driven. The power management one of the most challenging problems in such networks. Thus design of energy-aware collaborative signal processing algorithms is important in order to maximize the lifetime of such sensor networks.

In this paper, a collaborative tracking algorithm is developed for such a wireless ad-hoc sensor network consisting of randomly distributed low-end sensors. Every cluster of sensors is assumed to have a high-end sensor that acts as a data gathering node and communicate with all the sensors in its cluster to collect observations from them. Only this data gathering nodes is able to communicate with a base station and other data gathering node in the network. The proposed distributed collaborative algorithm is based on the total energy consumption of the sensor network, in which the active sensors at each time instant are chosen by a data gathering node in order to minimize the total energy spent by the network. The data gathering node decides the next set of active sensors based on a cost function calculated by taking into account energy requirements for communication and sensing.

\section{System Model}

We assume that the target movement can be modeled by a discrete-time, linear, dynamical system perturbed by additive noise. Let $\vec{x}\left(t-\frac{\tau(t)}{2}\right)$ be the state of the target at time $t$ which consists of the position and velocity. We can then model the target movement as,

$$
\vec{x}\left(\mathrm{t}-\frac{\tau(t)}{2}+\mathrm{T}\right)=\mathrm{A} \vec{x}\left(t-\frac{\tau(t)}{2}\right)+\vec{u}\left(t-\frac{\tau(t)}{2}\right)
$$

where $\vec{u}$ is the state noise which is assumed to be zero-mean with covariance matrix $\mathbf{Q}, \mathrm{T}$ is the sampling time and is the system matrix A.

The observation vector with $\mathrm{M}$ active sensors can be written as,

$$
\vec{y}\left(t-\frac{\tau(t)}{2}\right)=\left[\begin{array}{lllll}
\tau(t)_{1} & f_{d}(t)_{1} & \cdots \tau(t)_{m} & f_{d}(t)_{m}
\end{array}\right]^{T}
$$

where $\tau(t)_{m}$ and $f_{d}(t)_{m}$ are the time delay and Doppler measurement by the $\mathrm{m}^{\text {th }}$ active sensor. The observation model is then,

$$
\vec{y}\left(t-\frac{\tau(t)}{2}\right)=\mathrm{C} \vec{x}\left(t-\frac{\tau(t)}{2}\right)+\vec{v}\left(t-\frac{\tau(t)}{2}\right)
$$

where $\mathbf{C}$ is the observation matrix and $\vec{v}$ is the observation noise which is assumed to be zero-mean with covariance matrix $\mathbf{R}$.

We develop a distributed tracking algorithm based on the Kalman filter which recursively updates the estimate of the state vector by processing successive measurements. It should be noted that this Kalman filter is implemented distributively in that as active cluster changes the Kalman filter computation changes from one data gathering node to another. When the leadership is passed from one cluster to another cluster, the Kalman filter at the next leader sensor is 
initialized with the predicted state obtained from the previous leader sensor.

\section{Energy-Based Multi Sensor Collaboration}

The data gathering node chooses the set of active sensors at each time instant $t$ so that the total energy spent by the whole network is minimized. Let $S$ be the set of indices of all the sensors in the active cluster and $A$ be the set of indices of the current active sensors. Then the energy-based active sensor selection algorithm can be written compactly as:

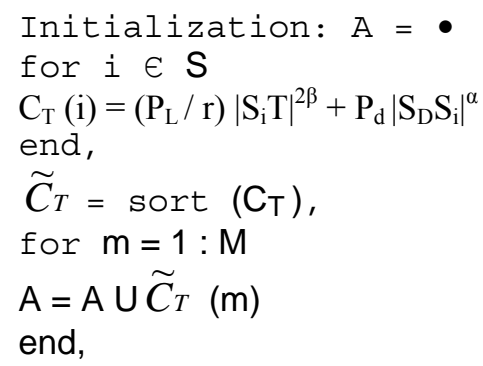

where $U$ denotes the union of sets , $\Phi$ is the empty set and $\mathrm{C}_{\mathrm{T}}$ total energy cost incurred by the whole network.

The total energy spent by the network at each instant is the sum of the sensing $\left(\mathrm{E}_{\text {sensing }}\right)$ and the communication $\left(\mathrm{E}_{\text {comm }}\right)$ energies:

$$
\mathrm{E}_{\text {Total }}=\mathrm{E}_{\text {sensing }}+\mathrm{E}_{\text {comm }}
$$

\section{Simulation results}

We simulate an ad-hoc wireless sensor network of an area $1000 \times 1000 \mathrm{~m}^{2}$ which is divided into 100 clusters of equal area. The entire sensor network 1000 randomly distributed low-end sensors and 100 data gathering nodes located at the center of each cluster. It is assumed that at each time instant only the M-active sensors in the network measure the time delay and the Doppler shift corresponding to the target. The sampling interval $\mathrm{T}$ is set to 1 and $\mathrm{M}=3$ in the following results.

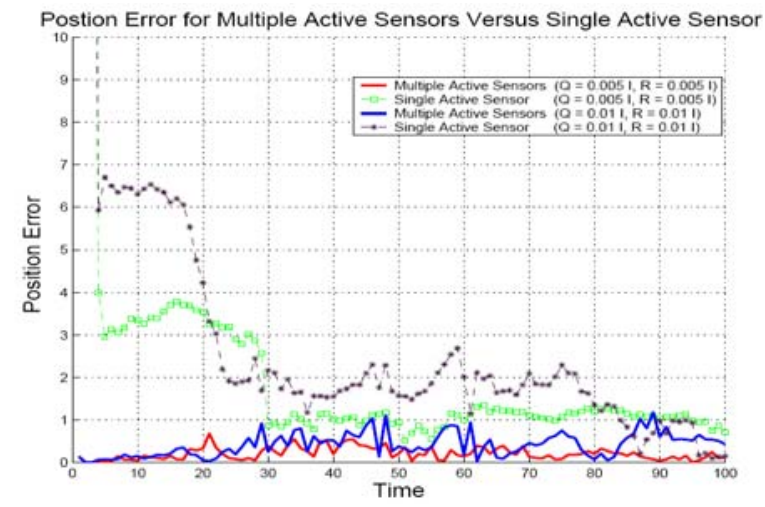

Fig.1. Target Position Error with Multiple Active Sensors versus Single Active Sensor.

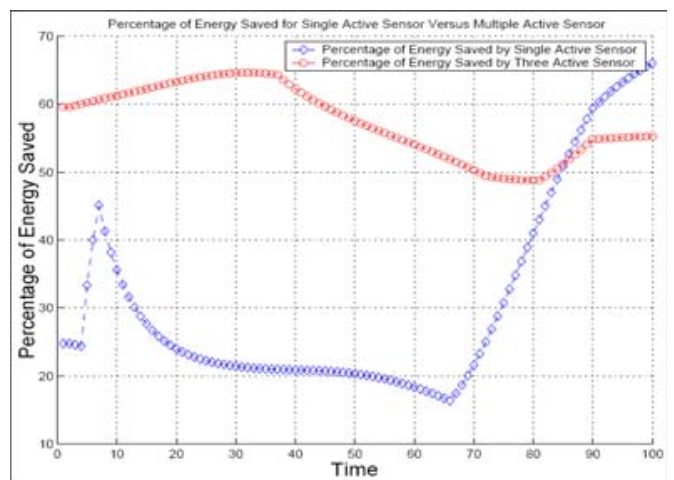

Fig.2. Percentage of Energy Savings for Single Active Sensor versus Multiple Active Sensors.

From Fig. 1, we can observe that the proposed energy efficient collaborative tracking algorithm with multiple active sensors can achieve smaller error values compared to a similar algorithm with only a single active sensor. From Fig. 2 we can see that about $48 \%-65 \%$ of energy is saved by using the multiple active sensors and $17 \%-66 \%$ of energy is saved by using a single active sensor.

\section{Conclusion}

In this paper, we presented an energy-aware collaborative tracking algorithm and a method to compute the achieved energy efficiencies for a wireless ad-hoc sensor network. The proposed energy-based distributed tracking algorithm attempts to reduce the total energy consumption of the whole network (as against that of a particular sensor) in order to maximize the life time of the total network.

\section{References}

[1] S. Balasubramanian, S. K. Jayaweera, and K. R. Namuduri, "Energy-Aware, Collaborative Tracking with Ad-Hoc Wireless Sensor Networks", in Proc. of Wireless Commun. and Networking Conf., New Orleans, LA, Mar. 2005. 